\title{
The Role of Ground-Based Robotic Observatories in Satellite Projects
}

\author{
R. Hudec ${ }^{1,2}$ \\ ${ }^{1}$ Astronomical Institute, Academy of Sciences, 25165 Ondrejov, Czech Republic \\ ${ }^{2}$ Faculty of Electrical Engineering, Czech Technical University in Prague, Technická 2, 16627 Prague 6, Czech Republic
}

Correspondence should be addressed to R. Hudec, rhudec@asu.cas.cz

Received 9 June 2009; Revised 25 August 2009; Accepted 20 December 2009

Academic Editor: Alberto J. Castro-Tirado

Copyright ( 2010 R. Hudec. This is an open access article distributed under the Creative Commons Attribution License, which permits unrestricted use, distribution, and reproduction in any medium, provided the original work is properly cited.

\begin{abstract}
We discuss the role of robotic telescopes in satellite projects, as well as related strategies. Most satellite projects in space astrophysics focus on high-energy astrophysics from X-rays to gamma-rays. A large fraction of objects of high-energy astrophysics emit also optical light, which is, in many cases, variable. The observation of these sources at optical wavelengths can provide valuable inputs for multispectral analysis of various categories of celestial high-energy (HE) sources. As the magnitudes of numerous objects are bright and can be hence accessed by robotic ground-based observatories, these observations can contribute to investigations and analyses of HE sources. We discuss in detail this possible contribution, with emphasis on the ESA INTEGRAL mission. In addition to this, there are also satellite projects outside the high-energy astronomy, in which the robotic telescopes can also play an important role. We will illustrate this on the example of the ESA satellite Gaia. In this project, robotic telescopes are expected not only to verify the triggers detected by satellite (such as transients and flares) but also to provide additional (mostly photometric) data for better scientific cases.
\end{abstract}

\section{Introduction}

Most satellite projects in space astrophysics focus on high energy astrophysics from X-rays to gamma-rays. A large fraction of objects of high-energy astrophysics emit also optical light, which is, in many cases, variable (Figure 1). The observation of these sources at optical wavelengths can provide valuable inputs for multispectral analysis of various categories of celestial high-energy (HE) sources. As the magnitudes of numerous objects are bright and can be hence accessed by (even small) robotic telescopes, these devices can effectively contribute to investigations and analyses of $\mathrm{HE}$ sources.

In addition to this, the robotic telescopes can also play an important role in satellite projects outside high-energy astrophysics. The astrometry mission Gaia of European Space Agency ESA can serve here as an example. As it will be shown later, very common is the situation when we have satellite (e.g., HE) monitoring data covering up to $\sim$ years, but we do not have simultaneous optical data. At the same time, the most important goal is to recognize active states of the sources (flares, high states, etc.) either to trigger the satellite observations, or, alternatively, to be able to concentrate on archival satellite data for those periods. In this aspect, robotic observatories can effectively contribute.

\section{The Role of Monitors}

Some types of astrophysical objects (e.g., blazars, cataclysmic variables, gamma-ray bursters, flare stars, etc.) exhibit rare flares for which satellite observations are important. These events cannot be monitored by satellites themselves in most cases. These events can be effectively monitored by ground based robotic telescopes (RT) generating $\mathrm{ToO}$ (Target of Opportunity) triggers for satellites with $\mathrm{ToO}$ regime.

The monitors, in contrast to alert telescopes, can deliver optical photometric data for objects prior and during the active/flaring states-wide-field (WF) coverage is important to cover as many sources as possible. There is a scientifically justified need to have this mode in robotic (i.e., autonomous remotely controlled) telescopes. RT with reasonably large 
$V$ fluxes - INTEGRAL sources OSA 5.1

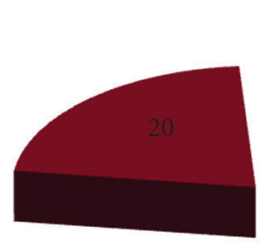

32
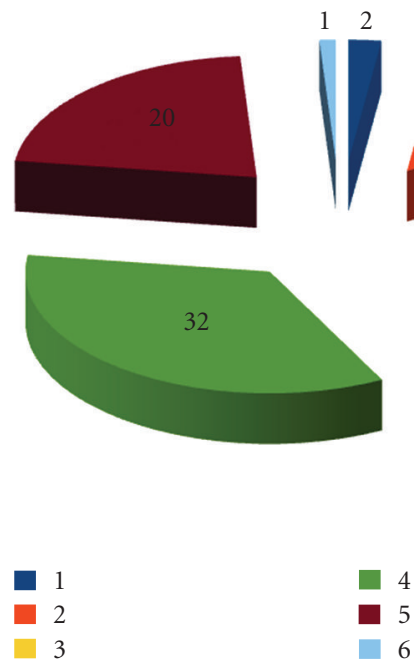

FIgure 1: The distribution of optical $V$ magnitudes of optically identified INTEGRAL gamma-ray sources. Most are brighter than mag 20, and more than half are brighter than mag 15. Legend dark blue $=\operatorname{mag} 2-6$, orange $=\operatorname{mag} 5-10$, yellow $=\operatorname{mag} 10-14$, green $=\operatorname{mag} 14-18$, purple $=\operatorname{mag} 18-22$, light blue $=\operatorname{mag} 22-26$. The numbers in the plot indicate number of objects with optical $V$ magnitude within the indicated range.

field of view (FOV), performing regular sky surveys, or with an attached WF camera, can serve as a monitoring device. In some cases, even post-flare monitoring is important as shown by magnetar candidate GRB070610 optical flares [1] in order to (1) detect the optical flares and (2) detect possible recurrence (this is a very difficult task, but important one, which can be performed only by robotic instruments as the recurrence cannot be predicted).

\section{The HE Sources as Optical Emitters}

The HE sources belong to both galactic as well as extragalactic sources. In the following subsections we will very briefly discuss both groups.

3.1. Galactic HE Sources. There are numerous categories of galactic HE sources, most important ones are listed below:

(1) Cataclysmic Variables (CVs) and related objects, example: GK Per,

(2) Low Mass X-ray Binaries (LMXRB), example: HZ Her $=\operatorname{Her} \mathrm{X}-1$,

(3) High Mass X-ray Binaries (HMXRB), example: Cyg $\mathrm{X}-1$,

(4) X-ray transients,

(5) New types of sources.

The fact that there are numerous CVs among the gamma-ray sources observed by the ESA INTEGRAL satellite (perhaps up to $10 \%$ of all INTEGRAL gamma-ray sources) represent one of interesting new findings over the last few years. Moreover, few symbiotic stars (SSs) were also identified with INTEGRAL gamma-ray sources.

3.1.1. Are the Optically Variable Galactic HE Sources "Variable Stars"? As many of the HE sources do have optical variable emission, a natural question arises, what is the link between these sources and classical variable stars (VS).

(1) Yes, some are X-ray and gamma-ray loud cataclysmic variables and symbiotic stars (SS) can serve as examples.

(2) Some of LMXRB, HMXRB are VS of "non-classical categories".

(3) Some are newly detected VS.

(4) Some are not VS such as the new category of galactic gamma-ray bursts (GRBs).

(5) The dominant role of CVs is obvious: The contribution of CVs to galactic X-ray background may be greater than assumed before, based on INTEGRAL results in hard X-rays (IBIS experiment).

3.2. Extragalactic HE Sources. Numerous celestial HE sources belong to the category of extragalactic sources; the most important types are listed below:

(1) AGN,

(2) Blazars,

(3) Optical Afterglows and Optical Transients of Gamma-Ray Bursts (GRBs),

(4) $\mathrm{SNe}$,

(5) LBV (Luminous Blue Variables in external galaxies). They are worth study as they can at some active states mimic the light behaviour of optical afterglows of GRBs.

Several examples of the blazars detected in gamma-rays by the INTEGRAL satellite are given and discussed later in this paper.

\section{Modes of Observations}

There are various modes of optical observations required for the HE sources. The situation is very complex, as the sources belong to various categories.

Satellite Campaigns. One of the most important modes of supporting optical ground-based observations is the response to satellite observing campaigns. While the satellite observation itself usually lasts for several days, the whole observing campaign lasts typically for weeks, as also the time interval before and after the satellite observation needs to be covered. Dense coverage during the satellite observation is required, with less dense coverage before and after. Magnitudes of the targets are typically 12-18 but occasionally can go deeper. Example: satellite campaigns organized by blazar observers. 
In addition to that, planned observations (mostly known in advance) of optically variable sources by satellites can be supplemented by optical ground based observations, with similar requirements as described above. Example: simultaneous optical observations for targets approved for Newton, Chandra, INTEGRAL.

Monitoring for Triggering Satellite (ToO-Target of Opportunity) Observations. They belong to another important type of optical observations of HE sources. In most cases, moderate sampling of $\sim 1$ point/day is enough. Magnitudes are typically 12-18. Example: ToO proposal on blazars within INTEGRAL project. Here, the blazars included in the approved list are to be optically monitored for possible brightening. Again, about 1 photometric point/day is acceptable for most of the sources.

Providing Optical Data for Non-Triggered Satellite Observations. They represent another category (e.g., providing optical monitoring data for the time span of INTEGRAL operation, i.e., 2002-2012). Typically 1 point/day (or even less) is enough. Magnitudes of most of the objects are typically within the range 10-18. This type of observations allows to compare behaviour of gamma-ray sources at various energies and is hence physically important as from such comparison physical interpretations and conclusions may be drawn. Example: Ondrejov D50 RT long-term monitoring of INTEGRAL CVs and blazars.

Alert Followup Observations. They represent another important type. They need fast response, better (but not necessarily) automated. However, even a site with non automated instrumentation has chance due to observational/weather constraints. Mostly Gamma-Ray Bursts (GRBs) belong to the group of objects observed this way, but occasionally various types of other flaring and transient targets may be added. Expected magnitudes are 6-22 but in some cases (optically dim GRBs) even fainter. With the ESA Gaia satellite, we expect another (Gaia project related) type of alerts, which will point on suspicious (mostly flaring) objects detected within Gaia. As the Gaia itself will have limited ability to confirm the reality of these objects, in most cases the final confirmation and further analyses of Gaia alert triggers will rely on ground based (preferably robotic) observers. Example: GRB followup.

Verifying Suggested Identifications. They represent another type of job where robotic observatories may contribute. Typical magnitudes are $10-20$. The preferred response is within days or a week. Photometry both with good sampling as well as moderate sampling, photometry with filters, spectroscopy (including low dispersion), are required. Example: confirmation of INTEGRAL CV candidates revealed by optical spectroscopy.

Optical Supplementary Analyses. Optical supplementary analyses of HE sources (for complex multispectral analyses) may also add valuable optical data for understanding the
TABLE 1: Cataclysmic variables detected in hard X-rays by INTEGRAL.

\begin{tabular}{|c|c|c|c|}
\hline \multicolumn{4}{|c|}{ Detected CVs } \\
\hline GCVS Name & RA (2000) & $\operatorname{DEC}(2000)$ & Object Type \\
\hline IGR J00234+6141 & $00: 22: 57.63$ & $+61: 41: 07.8$ & $\mathrm{dq}$ \\
\hline$\underline{\text { V709 Cas }}$ & $00: 28: 48.84$ & $+59: 17: 22.3$ & $\mathrm{dq}$ \\
\hline$\underline{\mathrm{XY} \text { Ari }}$ & 02:56:08.10 & $+19: 26: 34.0$ & $\mathrm{dq}$ \\
\hline GK Per & 03:31:12.01 & $+43: 54: 15.4$ & $\mathrm{na} / \mathrm{dq}$ \\
\hline$\underline{V 1062 \mathrm{Tau}}$ & 05:02:27.47 & $+24: 45: 23.4$ & $\mathrm{dq}$ \\
\hline$\underline{\text { TV Col }}$ & $05: 29: 25.52$ & $-32: 49: 04.0$ & $\mathrm{dq}$ \\
\hline$\underline{\text { IGR J05346 }}-\underline{5759}$ & $05: 34: 50.60$ & $-58: 01: 40.7$ & vy: \\
\hline$\underline{\text { BY Cam }}$ & 05:42:48.77 & $+60: 51: 31.5$ & $\mathrm{am}$ \\
\hline$\underline{\text { MU Cam }}$ & $06: 25: 16.18$ & $+73: 34: 39.2$ & $\mathrm{dq}$ \\
\hline 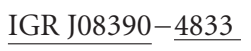 & 08:38:49.11 & $-48: 31: 24.7$ & $\mathrm{cv}$ \\
\hline XSS J12270- $\underline{4859}$ & $12: 27: 58.90$ & $-48: 53: 44.0$ & $\mathrm{dq}$ \\
\hline V834 Cen & 14:09:07.30 & $-45: 17: 16.2$ & $\mathrm{am}$ \\
\hline$\underline{\text { IGR J14536- }} \underline{5522}$ & $14: 53: 41.06$ & $-55: 21: 38.7$ & $\mathrm{dq}$ \\
\hline IGR J15094- $\underline{6499}$ & 15:09:26.01 & $-66: 49: 23.3$ & $\mathrm{dq}$ \\
\hline NY Lup & $15: 48: 14.59$ & $-45: 28: 40.5$ & $\mathrm{dq}$ \\
\hline 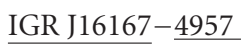 & $16: 16: 37.20$ & $-49: 58: 47.5$ & dq: \\
\hline$\underline{\text { IGR J16500- }} \underline{3307}$ & $16: 49: 55.64$ & $-33: 07: 02.0$ & $\mathrm{dq}$ \\
\hline V2400 Oph & $17: 12: 36.43$ & $-24: 14: 44.7$ & $\mathrm{dq}$ \\
\hline 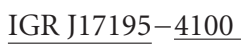 & $17: 19: 35.60$ & $-41: 00: 54.5$ & dq: \\
\hline$\underline{\text { IGR J17303 }}-\underline{0601}$ & $17: 30: 21.90$ & $-05: 59: 32.1$ & $\mathrm{dq}$ \\
\hline V2487 Oph & $17: 31: 59.80$ & $-19: 13: 56.0$ & na \\
\hline AM Her & $18: 16: 13.33$ & $+49: 52: 04.3$ & $\mathrm{am}$ \\
\hline$\underline{\text { IGR J18173 }}-\underline{2509}$ & $18: 17: 22.25$ & $-25: 08: 42.9$ & $\mathrm{cv}$ \\
\hline V1223 Sgr & 18:55:02.31 & $-31: 09: 49.6$ & $\mathrm{dq}$ \\
\hline 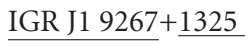 & 192627.03 & +132203.2 & $\mathrm{cV}$ \\
\hline V1432 Aql & 19:40:11.42 & $-10: 25: 25.8$ & $\mathrm{am}$ \\
\hline V2306 CYg & 19:58:14.48 & $+32: 32: 42.2$ & $\mathrm{dq}$ \\
\hline V2069 CYg & $21: 23: 44.84$ & $+42: 18: 01.8$ & dq: \\
\hline IGR J21335+ $\underline{5105}$ & $21: 33: 43.65$ & $+51: 07: 24.5$ & $\mathrm{dq}$ \\
\hline$\underline{\text { SS CYg }}$ & $21: 42: 42.80$ & $+43: 35: 09.9$ & ugss \\
\hline FO Aqr & $22: 17: 55.39$ & $-08: 21: 03.8$ & $\mathrm{dq}$ \\
\hline$\underline{\mathrm{AO} \text { Psc }}$ & 22:55:17.99 & $-03: 10: 40.0$ & $\mathrm{dq}$ \\
\hline
\end{tabular}

physics of the sources. The typical magnitudes are 1020. Again, photometry both with good sampling as well as moderate sampling, photometry with filters, and spectroscopy (including low dispersion) are required. Example: Investigation of gamma-ray loud blazars in optical light.

\section{Examples of Ground-Based Observations}

5.1. INTEGRAL Cataclysmic Variables. The ESA INTEGRAL satellite [2], launched in 2002 and expected to operate at least until 2012, with its 4 onboard telescopes for analyses of gamma-ray sources simultaneously in gamma-ray, X-rays, 
TABLE 2: IBIS observations of V1223 Sgr.

\begin{tabular}{|c|c|c|c|c|c|}
\hline $\mathrm{JD}(24 \ldots)$ & Exp. Time & $\begin{array}{c}\text { Flux }(15-25) \mathrm{keV} \\
{\left[10^{-12} \mathrm{erg} \mathrm{s}^{-1} \mathrm{~cm}^{-2}\right]}\end{array}$ & $\begin{array}{c}\text { Flux }(25-40) \mathrm{keV} \\
{\left[10^{-12} \mathrm{erg} \mathrm{s}^{-1} \mathrm{~cm}^{-2}\right]}\end{array}$ & $\begin{array}{c}\text { Flux }(40-60) \mathrm{keV} \\
{\left[10^{-12} \mathrm{erg} \mathrm{s}^{-1} \mathrm{~cm}^{-2}\right]}\end{array}$ & $\begin{array}{c}\text { Flux }(60-80) \mathrm{keV} \\
{\left[10^{-12} \mathrm{erg} \mathrm{s}^{-1} \mathrm{~cm}^{-2}\right]}\end{array}$ \\
\hline $52710.38-52752.01$ & 109.2 & $161.00 \pm 14.50$ & $57.90 \pm 4.88$ & $<4.93$ & $<6.26$ \\
\hline $52917.17-52926.84$ & 151.1 & $112.00 \pm 11.30$ & $51.10 \pm 4.19$ & $21.30 \pm 4.24$ & $<5.48$ \\
\hline $53082.07-53119.10$ & 228.1 & $127.00 \pm 8.90$ & $50.00 \pm 3.28$ & $23.10 \pm 3.48$ & $10.00 \pm 4.54$ \\
\hline $53267.41-53305.97$ & 134.5 & $126.00 \pm 12.50$ & $55.40 \pm 4.46$ & $25.40 \pm 4.75$ & $27.70 \pm 6.23$ \\
\hline $53440.61-53479.81$ & 90.9 & $155.00 \pm 15.20$ & $61.30 \pm 5.53$ & $24.10 \pm 5.85$ & $<7.69$ \\
\hline $53602.80-53672.88$ & 409.6 & $<7.17$ & $31.80 \pm 2.65$ & $<2.82$ & $<3.78$ \\
\hline $53781.06-53809.24$ & 282.1 & $132.00 \pm 10.00$ & $48.50 \pm 3.50$ & $13.90 \pm 3.56$ & $<4.69$ \\
\hline $52710.38-53809.25$ & 1405.5 & $103.00 \pm 3.90$ & $46.40 \pm 1.42$ & $15.10 \pm 1.48$ & $12.30 \pm 1.97$ \\
\hline
\end{tabular}

TABLE 3: List of blazars observed by the INTEGRAL satellite in hard X-rays.

\begin{tabular}{|c|c|c|c|c|c|c|}
\hline \multirow{2}{*}{$\frac{\text { Source }}{1 \mathrm{ES} 0647+250}$} & \multirow{2}{*}{$\frac{\mathrm{RA}}{065046.6}$} & \multirow{2}{*}{$\frac{\mathrm{DEC}}{+250300}$} & \multicolumn{2}{|c|}{ Gal coord. } & \multirow{2}{*}{$\frac{\mathrm{z}}{0.2030}$} & \multirow{2}{*}{$\begin{array}{c}\text { type } \\
\text { BL Lac }\end{array}$} \\
\hline & & & 190.283 & 10.996 & & \\
\hline PKS 0823-223 & 082601.5729 & -223027.204 & 243.990 & 8.930 & 0.9100 & Possible Q \\
\hline lES $2344+514$ & 234704.919 & +514217.87 & 112.892 & -9.908 & 0.0440 & BL Lac \\
\hline $8 \mathrm{C} 0149+710$ & 015325.8511 & +711506.463 & 127.920 & 8.983 & 0.0220 & Q \\
\hline 4C 47.08 & 030335.2422 & +471616.276 & 144.986 & -9.863 & 0.4750 & BL Lac \\
\hline $87 \mathrm{~GB} 02109+5130$ & 010328.1 & +432259 & & & & BL Lac \\
\hline BL Lac & 220243.2914 & +421639.980 & 92.590 & -10.441 & 0.0688 & BL Lac \\
\hline S5 $0716+714$ & 072153.4485 & +712036363 & 143.981 & 28.018 & 0.3000 & BL Lac \\
\hline S5 $0836+710$ & 084124.3653 & +70 5342.173 & 143.541 & 34.426 & 2.1720 & Q \\
\hline 3C 454.3 & 225357.7479 & +160853.561 & 86.111 & -38.184 & 0.8590 & Q \\
\hline 3С 279 & 125611.1665 & -054721.525 & 305,104 & 57.062 & 0.5362 & Q \\
\hline 3C 273 & 22906.6997 & +020308.598 & -11.010 & 4.380 & 0.1583 & Q \\
\hline PKS1830-211 & 183339.888 & -210339.77 & 12.166 & -5.712 & 2.5070 & Q \\
\hline $\operatorname{Mrk} 421$ & 110427.3139 & +38 1231.799 & 179.832 & 65.032 & 0.0300 & BL Lac \\
\hline J1656.3-3302 & 165619.2 & -330148 & 350.604 & 6.361 & & $?$ \\
\hline IGR J22517+2218 & 225153.498 & +221737.29 & 89.690 & -32.750 & 3.6680 & Q \\
\hline PKS0537-441 & 053843.5 & -440505 & 250.078 & -31.110 & 0.8960 & BL Lac \\
\hline $3 \mathrm{C} 66 \mathrm{~A}$ & 022239.6115 & +430207.799 & 140.143 & -16.767 & 0.4440 & Blazar \\
\hline Mrk 501 & 165352.2167 & +394536.609 & 63.600 & 38.859 & 0.0336 & BL Lac \\
\hline $1 \mathrm{ES} 2344+514$ & 234704.919 & +514217.87 & 112.892 & -9.908 & 0.0440 & BL Lac \\
\hline 1ES $1959+650$ & 195959.8521 & +650854653 & 98.003 & 17.670 & 0.0480 & Blazar \\
\hline
\end{tabular}

and (for brighter objects) optical $V$ band, is suitable for: (a) detection of the populations of CVs and symbiotics with the hardest X-ray spectra, (b) simultaneous observations in the optical and hard X-ray regions, and (c) long-term observations with Optical Monitoring Camera (OMC) including a search for rapid variations in observing series during science window (OMC observations also for systems bellow the detection limit in hard X-rays).

In total, $32 \mathrm{CVs}$ were detected (surprise, more than expected, almost $10 \%$ of INTEGRAL detections, see Table 1). $28 \mathrm{CVs}$ were seen by IBIS [6-8] —based on correlation of IBIS data and Downes CV catalogue [9]. 4 are CV candidates revealed by optical spectroscopy of IGR sources [10]—new CVs, not in Downes catalogue. They are mainly magnetic systems: 22 confirmed or propable intermediate polars (IPs), 3 polars, 2 dwarf novae, 4 probable magnetic CVs, 1 unknown. Periods: vast majority $P_{\text {orb }}>3 \mathrm{hr}$, that is, above the period gap (only one $<3 \mathrm{~h}$ ). 5 long period systems with $P_{\text {orb }}>7 \mathrm{hr}$. Some statistics: Intermediate polars represent only $\sim 2 \%$ of the catalogued CVs, but they dominate the group of CVs seen by IBIS. More such detections and new identifications can be hence expected. Many CVs covered remain unobservable by IBIS, but new have been discovered. IBIS tends to detect IPs and asynchronous polars: in hard $\mathrm{X}$-rays, these objects seem to be more luminous (up to the factor of 10) than synchronous polars (but detection of more CVs needed for better statistics). A few examples are listed and discussed below. 


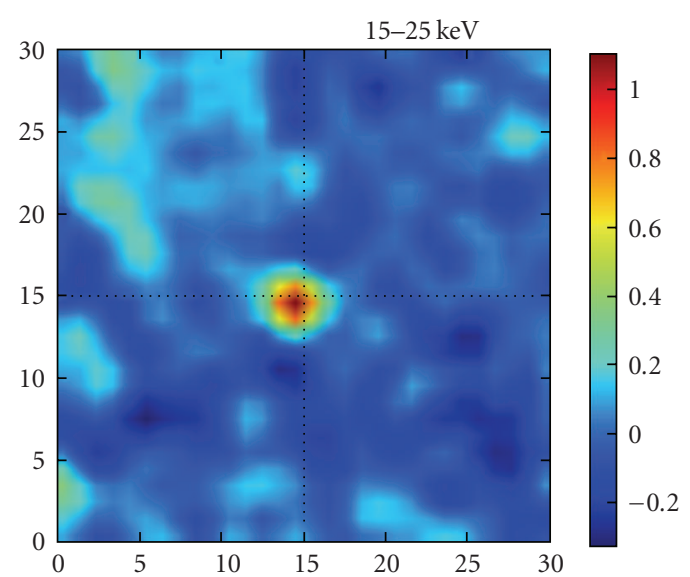

(a)

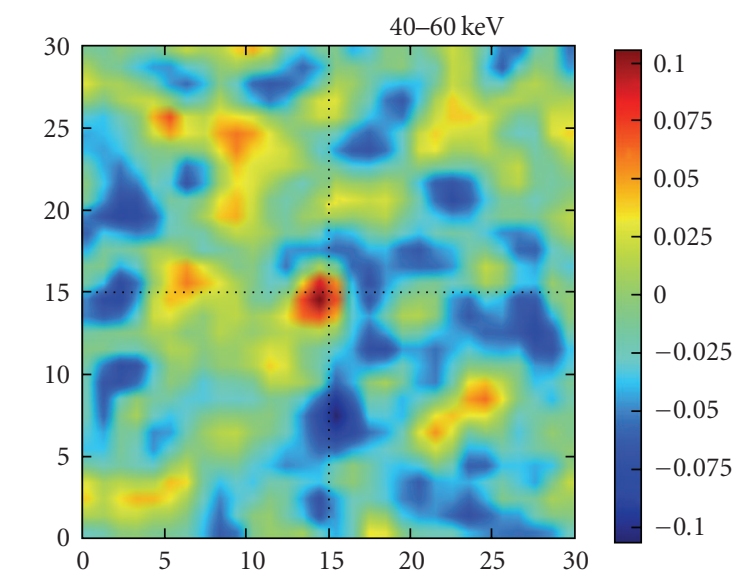

MJD 52729.191-53808.07

(c)

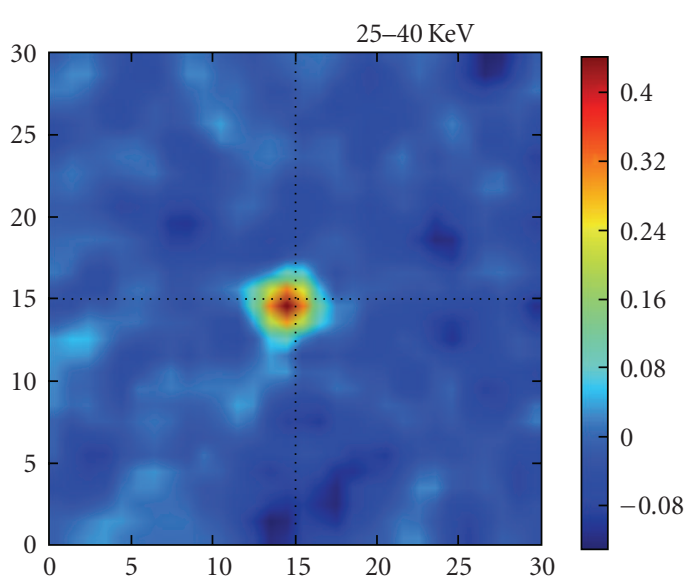

(b)

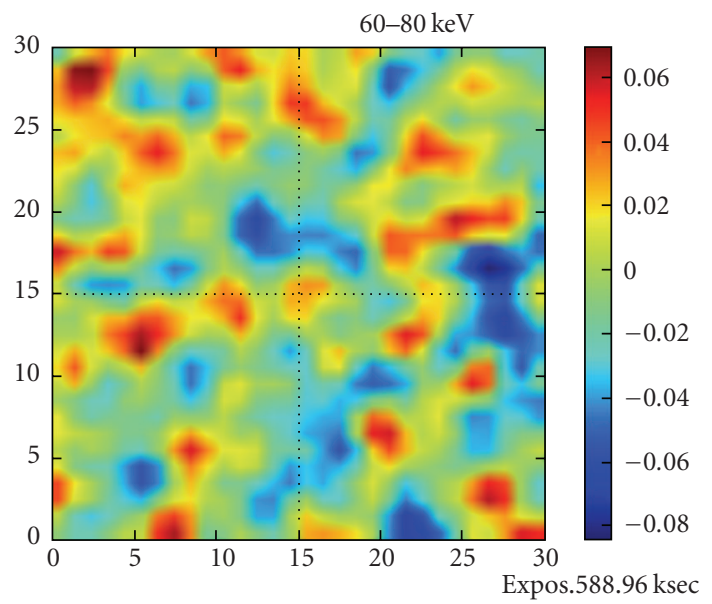

(d)

FIGURE 2: V1223 Sgr recent IBIS mosaics (coadded frames) all data (589 ks) intensity maps. The object is in the centre of the images.

V834 Cen. The optical light curve of V834 Cen during the lifetime of INTEGRAL shows active and inactive states. V834 Cen is a polar of AM Her class. This polar was probably detected by IBIS since it was in high (active, both optical and gamma-ray) state. This may explain why some CVs have been detected by IBIS and some not. Optical monitoring of sources is important as it can indicate active intervals when the object is expected to be active also in gamma-rays. However, comparing optical and gamma-ray activity is difficult in most cases due to lack of optical data. This is definitely a goal for robotic telescopes (example of monitoring of GK Per is shown in Figure 6).

V1223 Sgr. This object is an intermediate polar and represents the most significantly detected CV in the INTEGRAL IBIS survey, with a significance of 38 sigma in the $20-40 \mathrm{keV}$ final mosaic (Figures 2, 3, and 4, Table 2). Accretion via disk, bright X-ray source $(4 \mathrm{U} 1849-31)$. Orbital period: $\mathrm{P}_{\text {orb }}=$ $3.37 \mathrm{~h}[11,12]$. Rotational period of the white dwarf: $P_{\text {rot }}=$ $746 \mathrm{sec}$ [12]. Beat period (combined effect of $P_{\text {orb }}$ and $P_{\text {rot }}$ ): $P_{\text {beat }}=794.3 \mathrm{sec}[13]$.
Prominent long-term brightness variations: (i) outburst with a duration of $\sim 6 \mathrm{hr}$ and amplitude $>1 \mathrm{mag}$ [14], (ii) episodes of deep low state (decrease by several magnitudes) [15]. The object exhibits high-energy flaring activity: seen by IBIS (flare lasting for $\sim 3.5 \mathrm{hrs}$ during revolution 61 (MJD 52743), peak flux $\sim 3$ times of the average [6]. Analogous flares were seen also in optical (but at other times) by ground-based instrumentation (duration 6-24 hrs [14]). This confirms the importance of OMC instrument onboard INTEGRAL: even with $V \lim$ mag 15, it can provide valuable optical simultaneous data to gamma-ray observations.

Similar flares are known also for another IPs in optical, but not in soft gamma rays: Example TV Col [3], where 12 optical flares have been observed so far, five of them on archival southern sky patrol plates from the Bamberg Observatory (Figure 5). TV Col is an intermediate polar and the optical counterpart of the X-ray source 2A0526-328 [16]. TV Col is the first cataclysmic variable (CV) discovered through its X-ray emission. Recently TV Col was detected by INTEGRAL IBIS as a hard X-ray source [8]. Physics of the outbursts in IPs is either disk instability or an increase in mass transfer from the secondary. 


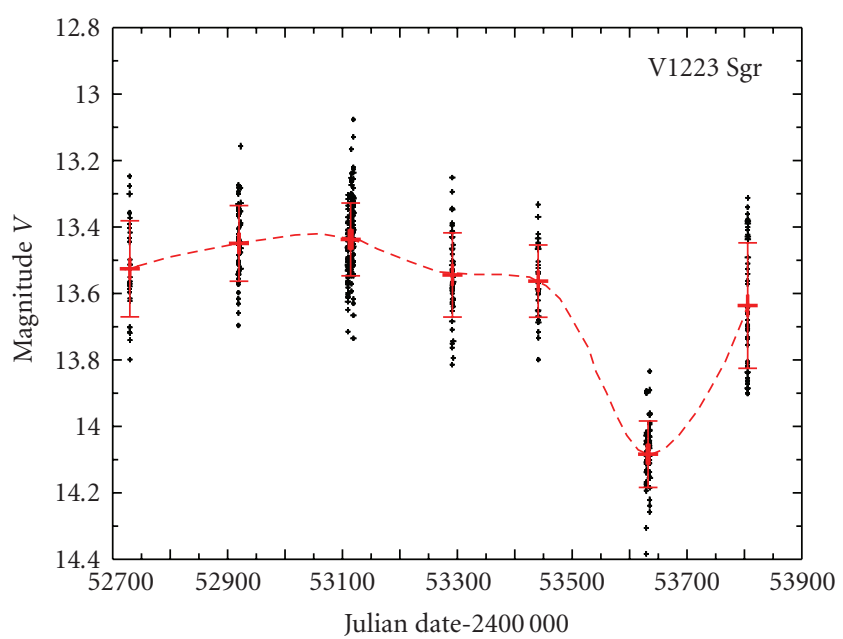

(a)

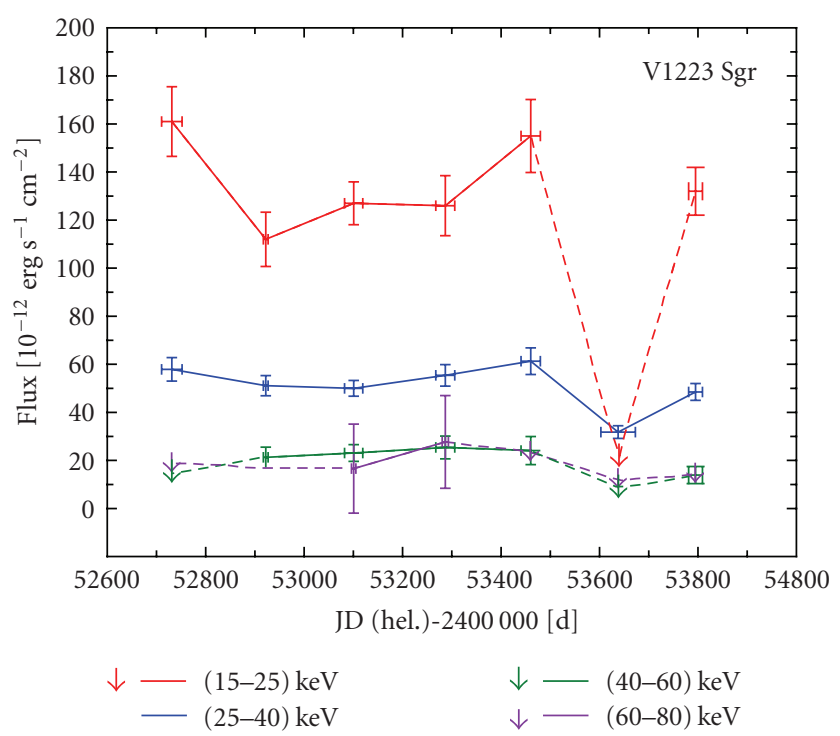

(b)

FIgUre 3: The X-ray (IBIS, (a)) and optical (OMC, (b)) light curves for V1223 Sgr. The fluxes especially in (15-25) keV and (25-40) $\mathrm{keV}$ bands are long-term variable with significant drop around MJD 53650. Optical variations are correlated with the changes in (15-25) keV, (25-40) keV and (40-60) keV spectral bands with correlation coefficient $0.81,0.82$, and 0.89 , respectively. The fluxes from INTEGRAL/JEM-X were persistent within their errors in monitored time period.

5.2. INTEGRAL Blazars. From the extragalactic HE sources, blazars belong to the most important and also optically violently variable objects. In Table 3 , we list a few examples of blazars analyzed with INTEGRAL observations.

$1 E S 1959+650$. This blazar is a gamma-ray loud variable object visible by IBIS in 2006 only, invisible in total mosaics and/or other periods. The optical light curve available for this light curve confirms the relation of active gamma-ray and active opticalstate (Figures 7 and 8).

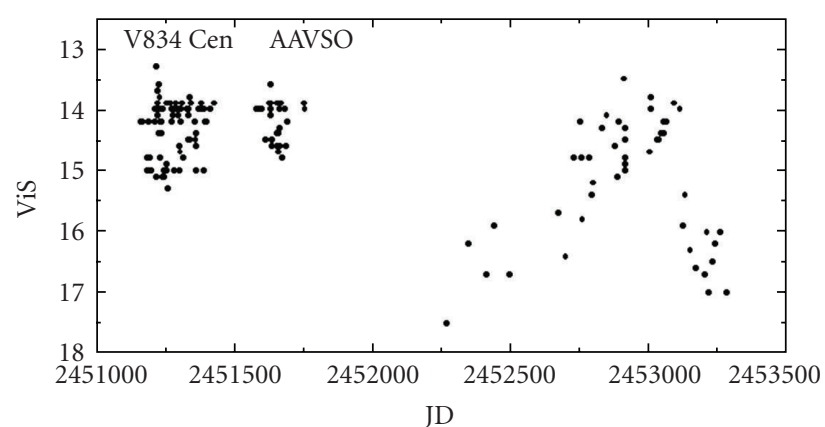

FIgUre 4: The AAVSO light curve for V834, a cataclysmic gammaray loud variable detected by the ESA INTEGRAL satellite. The active state around JD 2453000 represents the time period when the source was detected in gamma-rays, while it remained undetected at times when the source was in the inactive state.

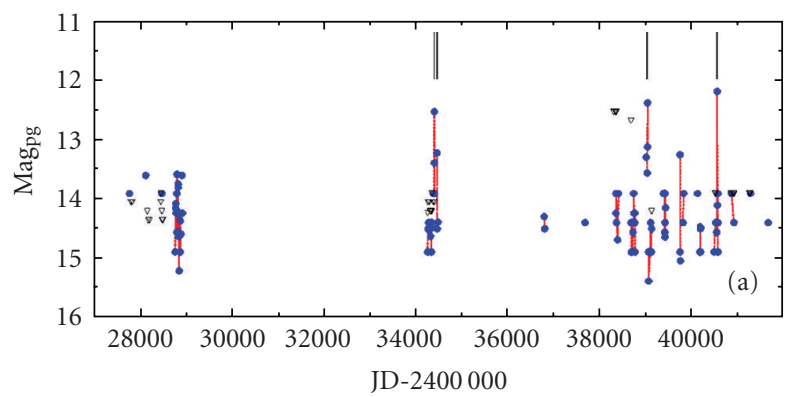

FIGURE 5: Long-term optical light curve of TV Col [3], where 12 optical flares have been observed so far, five of them on archival plates from the Bamberg Observatory. TV $\mathrm{Col}$ is an intermediate polar and the optical counterpart of the X-ray source 2A0526-328. This is the first cataclysmic variable $(\mathrm{CV})$ discovered through its Xray emission. Recently detected also by INTEGRAL IBIS.

3C66A. This blazar is visible by IBIS gamma-ray imager onboard INTEGRAL only during the optical flare shown below and is invisible other times (Figures 9 and 10). This confirms the importance of monitoring of the object in the optical light.

5.3. Identification and Classification of HE Sources. The RT can also serve as a effective tool in identification and classification of HE sources by optical monitoring and consequent detailed optical analyses of the error box content. Many of the HE are optically variable and hence can be identified (and classified) by their optical variability (Figure 11).

5.4. Poorly Understood Objects. In addition to the main types of HE objects described above, occasionally also objects worth study of another category appear. The variable objects at positions of Ultra High-Energy (UHE) sources can serve as an example, for example, the puzzling poorly investigated variable star at position of UHE source, namely the variable M6 star V347 Aql, with coordinates J2000.0 ICRS position of 


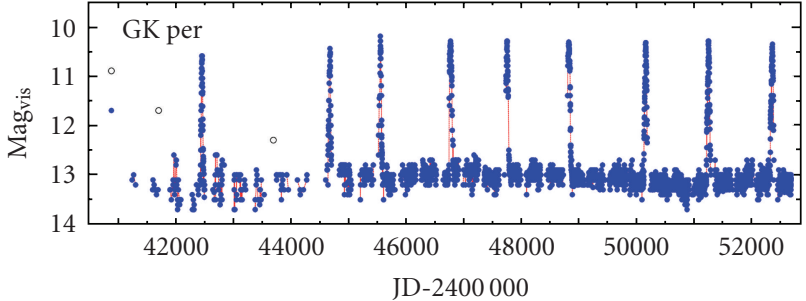

(a)

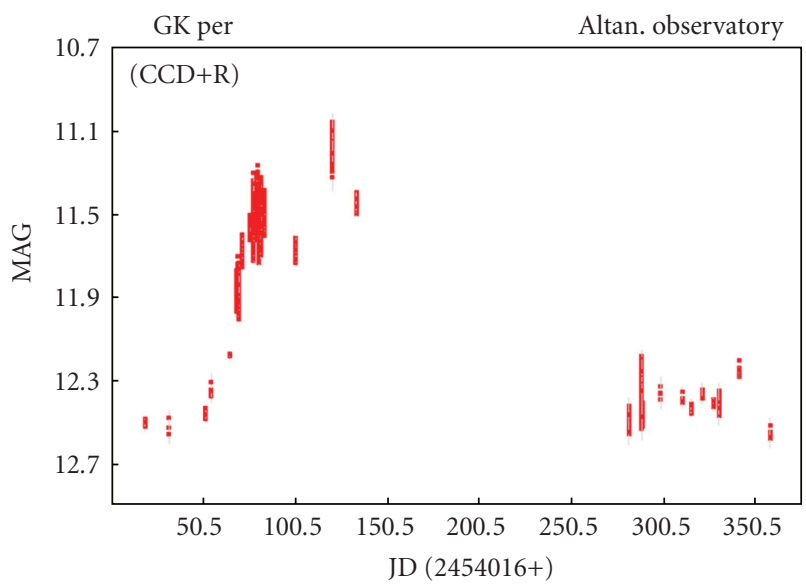

(b)

Figure 6: The long-term light curve of intermediate polar and INTEGRAL source GK Persei (top, source AAVSO) and the recent optical outburst in 2006 (bottom, according to Brat et al. 2006 [4]). The 2006 outburst appeared only 755 days from last outburst. For previous outbursts in 1992, 1996, 1999, 2002, and 2004, the separation was between 1091 and 1333 days, but before that the outburst separation was roughly $N \times 400 \mathrm{~d}, N=1,2,3$ [5].

$\mathrm{RA}=19 \mathrm{~h} 08 \mathrm{~m} 01.3 \mathrm{~s}, \mathrm{DEC}=+06 \mathrm{~d} 18^{\prime} 27$, and magnitude $B=11.5 \mathrm{mag}$. The star is within the error box of the new VHE (very high energy) source HESS J1908+063. The nature of this star (which is also an IRAS source) is unknown, previous possible classifications were a possible T Tauri star, or an oxygen rich irregular variable star.

The light curve is unknown, albeit the object magnitude amounts to $\mathrm{B} \sim 11$, that is, the star is a good target for small robotic observatories (Figure 12).

5.5. New Types of Optically Variable Objects. There are also newly detected types of optically variable HE sources. The optical counterpart of GRB070610/SWIFT J195509+261406 may serve as an example $[1,17]$. The basic parameters of this GRB are as follows: detected on 10 June 2007 20:52:26 UT by Swift/BAT as a normal burst [18], T90 $=4.6 \mathrm{~s}$, Photon index $1.76 \pm 0.25$, Fluence $(2.4 \pm 0.4) \cdot 10^{-7} \mathrm{erg} / \mathrm{cm}^{2}$ [19], XRT detected an X-ray counterpart 3100 s later [20] with a column density consistent with the Galactic. Pagani et al. [21] reported the detection of a variable optical counterpart, de Ugarte Postigo et al. [22] confirmed the detection with observations from the $1.5 \mathrm{~m}$ OSN. Kann et al. [23] suggested a Galactic origin, based on unusual flaring activity and

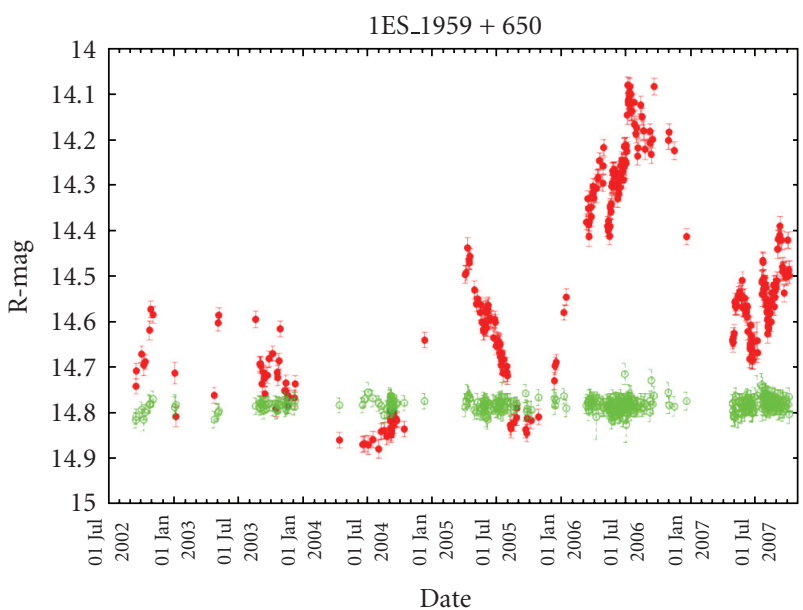

FIgure 7: Optical light curve of blazar 1ES 1959+650 (in red, Tuorla Observatory blazar monitoring program). The green symbols represent the control measurement of constant star.

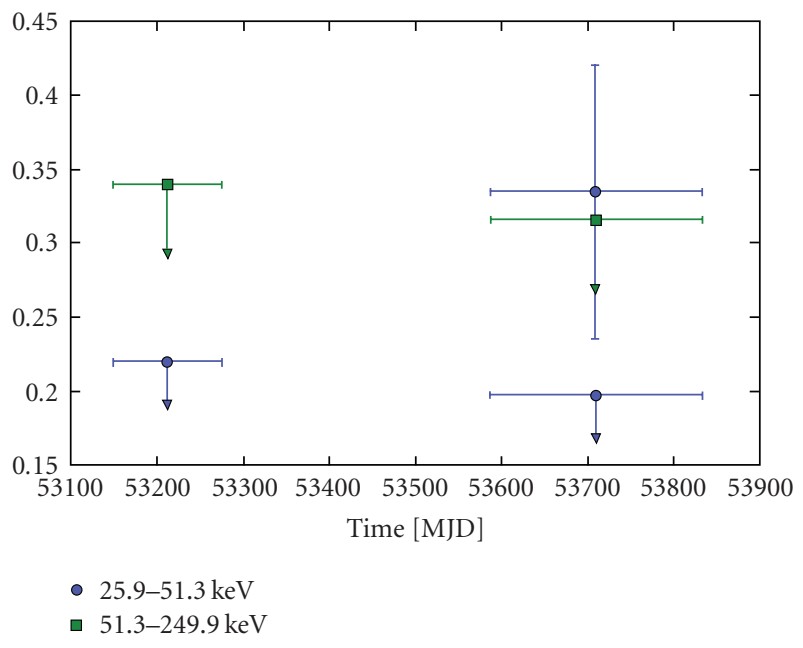

FIGURE 8: IBIS gamma-ray light curve of 1ES 1959+650. Blazar is in INTEGRAL gamma-ray imager IBIS visible only in data set corresponding to optical flare.

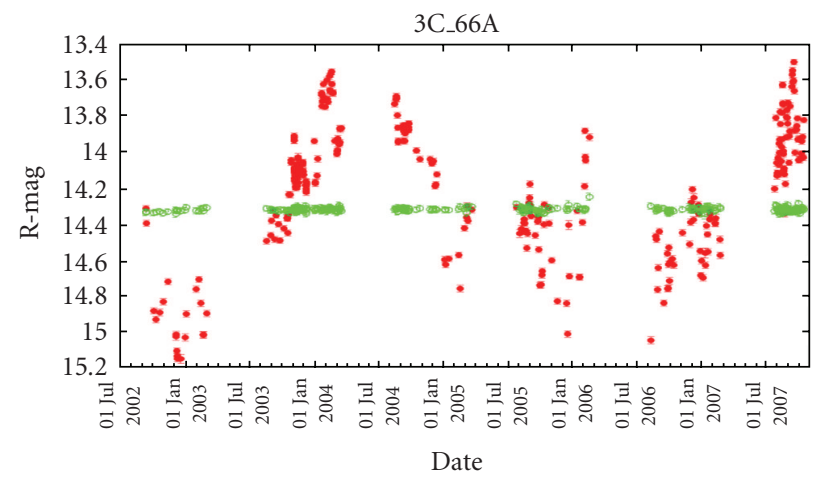

Figure 9: Optical light curve of blazar 3C66A (in red, Tuorla Observatory blazar monitoring program). The green symbols represent the control measurement of constant star. 


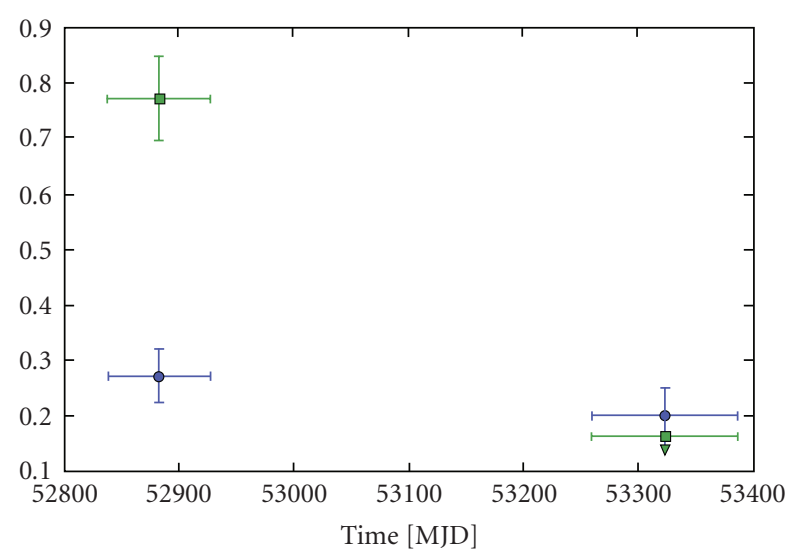

- $25.9-51.3 \mathrm{keV}$

- $51.3-249.9 \mathrm{keV}$

FiguRe 10: IBIS gamma-ray light curve of blazar 3C66A.

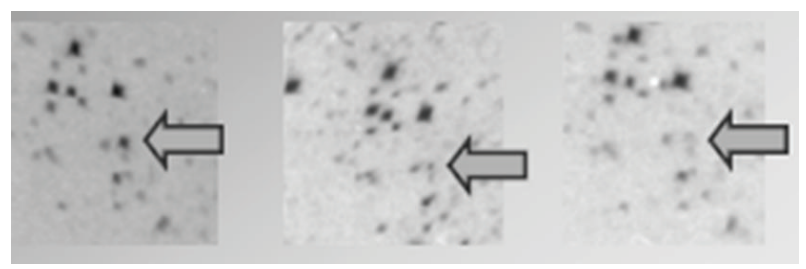

FIGURE 11: An example of appearance of INTEGRAL sources in optical light as variable objects. IGR J12349-6434 = RT Cru (hard X-ray symbiotic star) in optical light on Leiden Franklin Adams Plates. The object exhibits violent (amplitude 3 magnitudes) optical brightness variations.

location near the galactic plane: $l=63.3^{\circ}, b=-1^{\circ}$. About 40 optical flares peaking at up to I mag 14 as shown on Figure 13. The emission between flares slowly decreased until it disappeared with no detectable quiescent source.

\section{Supplementary Optical Data for Non-HE Satellites}

Another important role of robotic telescopes is in satellite projects outside high-energy astrophysics. The ESA Gaia project may serve as an example. Albeit its main goal is the ultra precise astrometry, Gaia will monitor all celestial objects down to magnitude 20 over a 5 years' time period. However, the photometric sampling will not be optimal; hence the supplementary observations provided by groundbased robotic telescopes are expected to provide a valuable contribution. The main goal of these supplementary observations are as follows: (1) confirm triggers (e.g., optical transients, flares, brightenings, etc.) detected by Gaia satellite and (2) provide additional photometric data with more dense sampling than provided by the satellite. The HE objects such as LMXRB, HMXRB, and Optical Afterglows and Optical Transients of GRBs can serve here as an example, together with various types of cataclysmic variables including SNe.

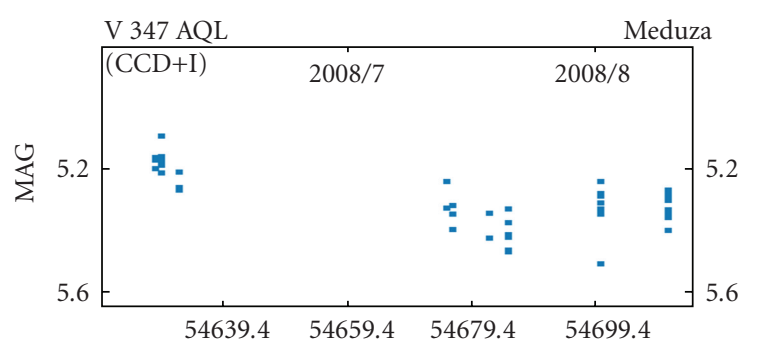

(a)

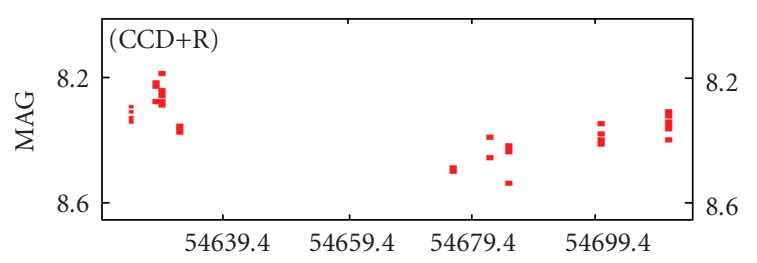

(b)

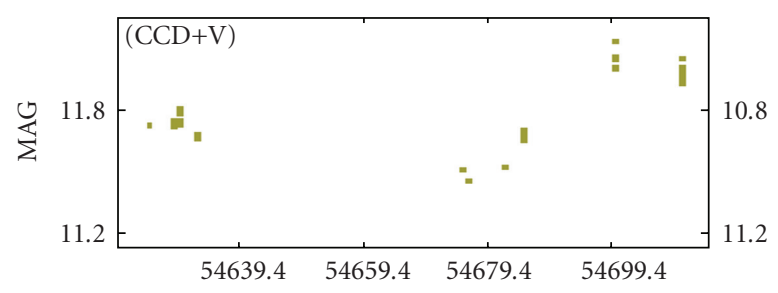

(c)

FIgURE 12: The VRI optical light curve V 347 Aql = HESS J1908+063 provided by the Altan Observatory, Czech Republic. The object exhibits both light changes as well as colour variation.

The peculiarity of ESA Gaia, where a substantial fraction of data will be as ultra-low dispersion spectra, raises a question about the role of focal devices with dispersive elements, that is, on a spectral alternative. This is fully scientifically justified, as the spectral type of Cepheids, Miras \& Peculiar Stars is known to change significantly with time. For example, all classical Cepheids definitely vary their spectral types. At maximum, they all have types around F5-F8. At minimum, the longer the period, the later is the spectral type (to K2) [24]. The long-term behaviour of spectral types of various variable celestial objects (so far only poorly investigated) may be a significant goal not only for ESA Gaia, but also for robotic ground-based optical telescopes equipped with corresponding dispersive elements.

\section{Supplementary Optical Data for CTA}

The CTA (Cherenkov Telescope Array), albeit not being a satellite, is in many aspects similar to satellite projects (see also the CTA effort at INTEGRAL Science Data Centre ISDC). There is a need for robotic monitoring of VHE $(\mathrm{TeV})$ sources, and alert system for $\mathrm{TeV}$ flaring triggers, for example, 1ES1959+650 ( $\mathrm{TeV}$ blazar at $z=0.047)$. There was an essential progress in Cherenkov telescopes over past years, and nowadays these deliver data analogous to what is known from other energy pass bands (i.e., images and 


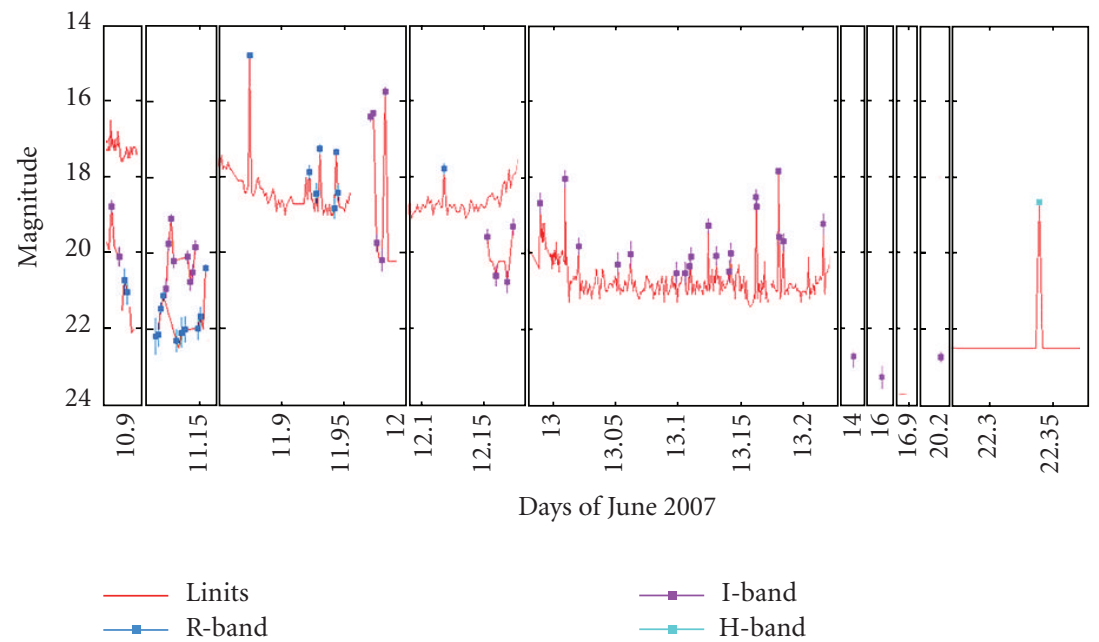

FIgURE 13: Optical light curve of flaring counterpart of GRB070610.

light curves). We propose to implement in Cerenkov systems analogous alert system as those currently used on satellites (i.e., rapid alerts sent to optical and radio observers). But also the opposite way may be essential, as the optical robotic telescopes can monitor $\mathrm{TeV}$ sources and report detected flares and active periods to Cherenkov teams.

\section{Conclusions}

The HE objects in many cases exhibit optical (and mostly variable emission) accessible in some cases even by small robotic observatories. For many of these sources there is a lack of optical data. The optical data provided by automated ground-based optical telescopes are important for multispectral analyses of the sources, contributing of better understanding of related physical processes. Even small apertures may contribute as some sources are brighter than magnitude 12 . In addition to that, robotic telescopes may play an important role also in satellite projects outside HE astrophysics, as shown on the example of the ESA Gaia, namely as devices confirming the satellite triggers, as well as delivering additional well sampled photometric data for particular objects.

\section{Acknowledgments}

The analyses of HE sources by the ESA INTEGRAL satellite were supported by ESA PECS project no. 98023, and in optical by grant 205/08/1207 provided by Grant Agency of the Czech Republic. The investigation of high-energy sources and cataclysmic variables by the ESA Gaia satellite is supported by the ESA PECS Project no. 98058. The analyses of GRBs in optical light including robotic telescopes are supported by the grant of the Grant Agency of the Czech Republic, 102/09/0997. The investigations of the Bamberg Observatory archival plates are supported by DAAD-AVCR project DAAD-25-CZ4/08 and the analyses of PARI plates by MSMT KONTAKT ME09027.

\section{References}

[1] A. J. Castro-Tirado, A. de Ugarte Postigo, J. Gorosabel, et al., "Flares from a candidate Galactic magnetar suggest a missing link to dim isolated neutron stars," Nature, vol. 455, no. 7212, pp. 506-509, 2008.

[2] C. Winkler, T. J.-L. Courvoisier, G. Di Cocco, et al., "The INTEGRAL mission," Astronomy and Astrophysics, vol. 411, no. 1, pp. L1-L6, 2003.

[3] R. Hudec, V. Simon, and J. Skalicky, "The astrophysics of cataclysmic variables and related objects," in Proceedings of ASP Conference, J.-M. Hameury and J.-P. Lasota, Eds., vol. 330, p. 405, San Francisco, Calif, USA, 2005.

[4] L. Brat, J. Strobl, P. Kubanek, et al., "Old nova GK Per in dwarf nova-type outburst," The Astronomer's Telegram, vol. 965, 2006.

[5] R. Hudec, "Optical properties of X-ray stars. I - X-ray system GK Per A $0327+43$. II- the secular brightness variations of the $\mathrm{X}$-ray system V 818 Sco/Sco X-1," Bulletin of the Astronomical Institute of Czechoslovakia, vol. 32, no. 2, pp. 93-120, 1981.

[6] E. J. Barlow, C. Knigge, A. J. Bird, et al., " $20-100 \mathrm{keV}$ properties of cataclysmic variables detected in the INTEGRAL/IBIS survey," Monthly Notices of the Royal Astronomical Society, vol. 372, no. 1, pp. 224-232, 2006.

[7] A. J. Bird, A. Malizia, A. Bazzano, et al., "The third IBIS/ISGRI soft gamma-ray survey catalog," The Astrophysical Journal, vol. 170, no. 1, pp. 175-186, 2007.

[8] R. Gális, D. Eckert, S. Paltani, F. Münz, M. Kocka, and R. Hudec, "Hard X-ray emission of cataclysmic variables observed by INTEGRAL," in Proceedings of the 7th INTEGRAL Workshop, Copenhagen, Denmark, September 2008.

[9] R. A. Downes, R. F. Webbink, M. M. Shara, H. Ritter, U. Kolb, and H. W. Duerbeck, "A catalog and atlas of cataclysmic variables: the living edition," Publications of the Astronomical Society of the Pacific, vol. 113, no. 784, pp. 764-768, 2001.

[10] N. J. Masetti, A. Malizia, A. Dean, A. Bazzano, and R. Walter, "Optical spectroscopic identification of candidate counterparts to four INTEGRAL sources," The Astronomer's Telegram, vol. 957, 2006.

[11] F. Jablonski and J. E. Steiner, "On the stability of the 13.2 minute oscillation of V1223 Sagittarii," The Astrophysical Journal, vol. 323, pp. 672-677, 1987. 
[12] J. P. Osborne, R. Rosen, K. O. Mason, and K. Beuermann, "New EXOSAT results from the intermediate polar V1223 SGR," Space Science Reviews, vol. 40, no. 1-2, pp. 143-149, 1985.

[13] J. E. Steiner, D. A. Schwartz, F. J. Jablonski, I. C. Busko, M. G. Watson, and J. P. Pye, "Identification of 4U 1849-31 with V1223 Sagittarii and discovery of optical pulsations," The Astrophysical Journal, vol. 249, pp. L21-L24, 1981.

[14] S. van Amerongen and J. van Paradijs, "Detection of a brief outburst from the intermediate polar V 1223 SGR," Astronomy and Astrophysics, vol. 219, no. 1-2, pp. 195-196, 1989.

[15] P. Garnavich and P. Szkody, "Observed low states in DQ Herculis systems," Publications of the Astronomical Society of the Pacific, vol. 100, pp. 1522-1528, 1988.

[16] B. A. Cooke, M. J. Ricketts, T. Maccacaro, et al., "The Ariel $\mathrm{V} / \mathrm{SSI} / \mathrm{catalogue}$ of high galactic latitude /absolute value of B greater than $10 \mathrm{deg} / \mathrm{X}$-ray sources," Monthly Notices of the Royal Astronomical Society, vol. 182, pp. 489-515, 1978.

[17] A. de Ugarte Postigo, A. J. Castro-Tirado, J. Gorosabel, et al., "GRB 070610: Flares from a peculiar Galactic," in GAMMARAY BURSTS 2007: Proceedings of the Santa Fe Conference, vol. 1000 of AIP Conference Proceedings, pp. 337-341, Santa Fe, NM, USA, 2008.

[18] C. Pagani, et al., "GRB Coordinates Network, Circular Service," 6489, 1, 2007.

[19] J. Tueller, et al., "GRB Coordinates Network, Circular Service," 6491, 1, 2007.

[20] C. Pagani, et al., "GRB Coordinates Network, Circular Service," 6490, 1, 2007.

[21] C. Pagani, et al., "GRB Coordinates Network, Circular Service," 6492, 1, 2007.

[22] A. de Ugarte Postigo, et al., "GRB Coordinates Network, Circular Service," 6501, 1, 2007.

[23] D. A. Kann, et al., "GRB Coordinates Network, Circular Service," 6505, 1, 2007.

[24] N. Samus, et al., Private communication, 2008. 

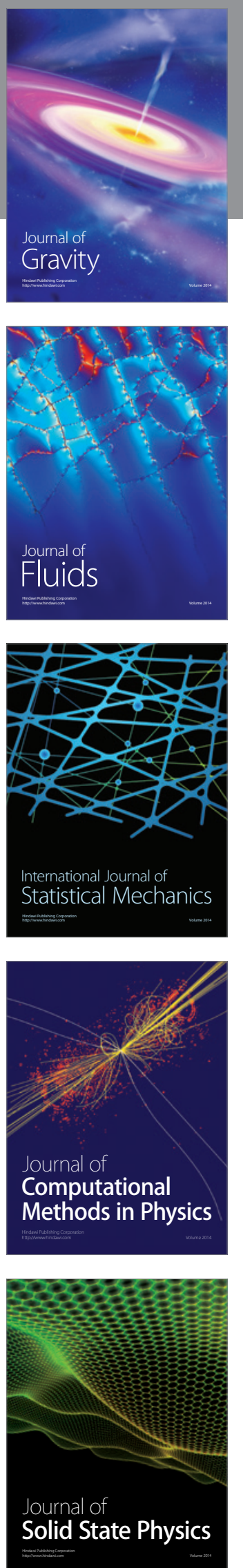

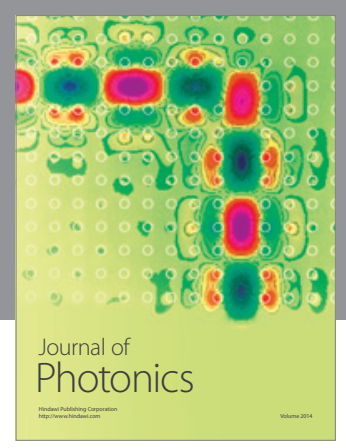

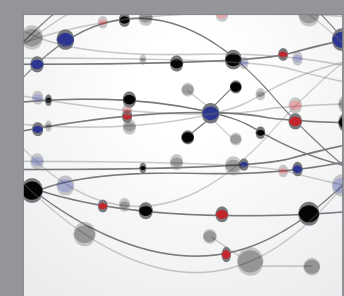

The Scientific World Journal
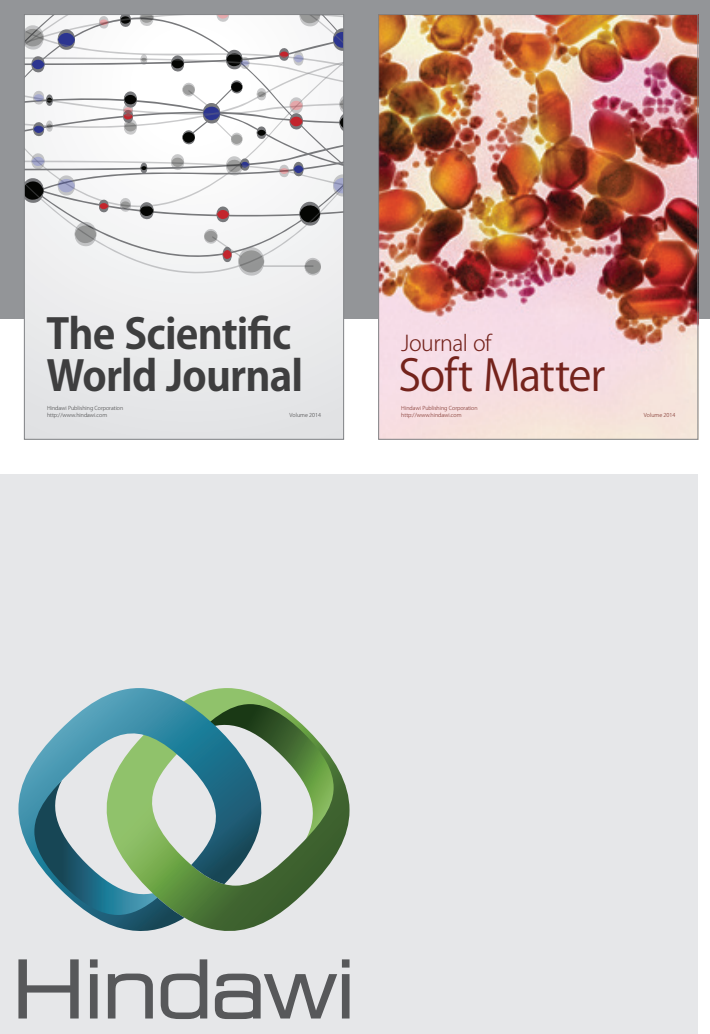

Submit your manuscripts at

http://www.hindawi.com
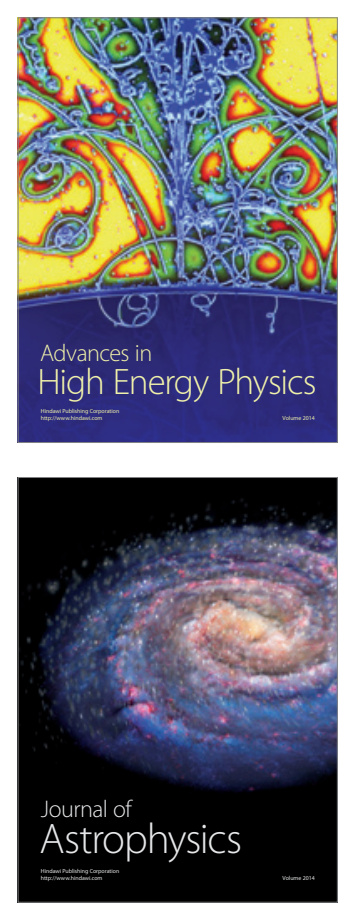
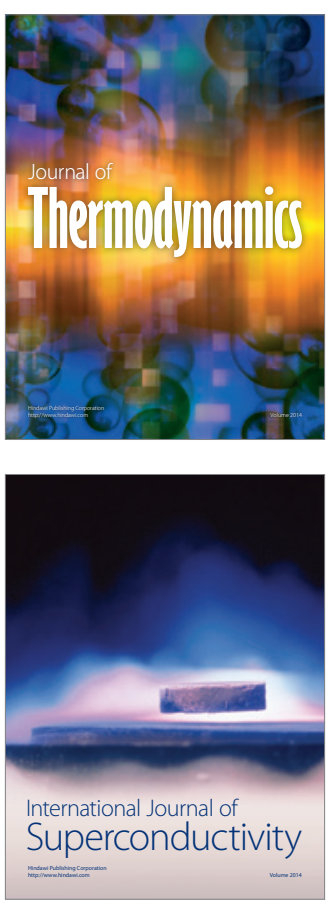
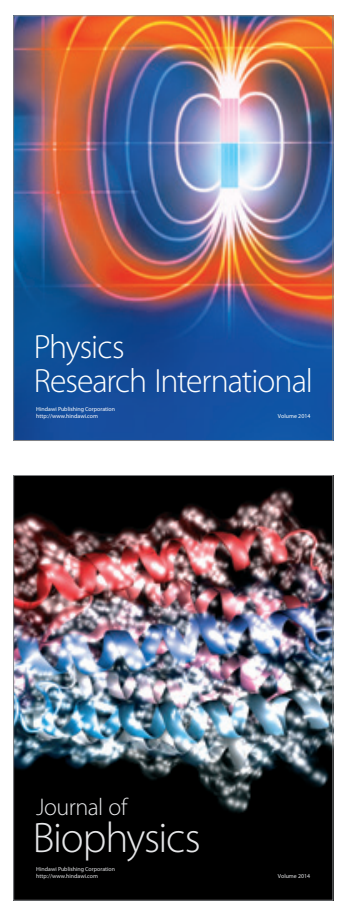
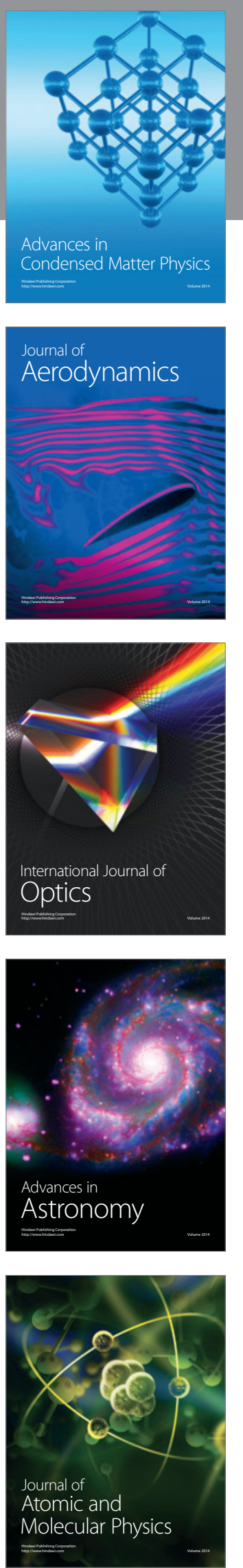